\title{
Epileptogenicity of a patient with the association of medullary venous malformation and cavernous malformation
}

\author{
Ataru Nishimura ${ }^{1}$, Takato Morioka ${ }^{1}$, Kimiaki Hashiguchi ${ }^{1}$, \\ Masahiro Mizoguchi $^{1}$, Shinji Nagata ${ }^{1}$, Satoshi O Suzuki ${ }^{2}$, Tomio Sasaki ${ }^{1}$ \\ ${ }^{1}$ Department of Neurosurgery, Graduate School of Medical Sciences, Kyushu University, Japan \\ ${ }^{2}$ Department of Neuropathology, Graduate School of Medical Sciences, Kyushu University, Japan
}

Key words: Cavernous malformation, Medullary venous malformation, Epileptogenisity

Received January 20, 2008; Accepted April 23, 2008; Published online May 9, 2008

\begin{abstract}
We present a surgical case of a 53-year-old man with lesion-related epilepsy associated with medullary venous malformation (MVM) and cavernous malformation (CM) in the right frontal lobe. He had frequent secondary generalized seizures since developing a MVMrelated intracerebral hemorrhage in the right frontal lobe at the age of 44 . Intraoperative electrocorticography demonstrated frequent paroxysmal activities on the yellowish cortex above the CM. Anterior frontal lobectomy intributaries was performed. The patient became seizure free after surgery. Pathologically, the irritative cortex above the CM had hemosiderin deposits and astrogliosis. The CM was surrounded by dilated veins of various sizes, indicating MVM, which confirmed the radiological findings of so-called caput medusae. Epileptogenicity in patients with CM and MVM is thought to be due to repeated hemorrhage from the CM. Surgical strategy is discussed.
\end{abstract}

cluding the irritative cortex, CM, MVM and its 


\section{Introduction}

Intracranial vascular malformations were classified by Russell and Rubinstein [1] into four types: arteriovenous malformation (AVM), cavernous angioma (cavernous malformation; CM), venous angioma (medullary venous malformation; MVM) and capillary telangiectasia. Among vascular malformations, AVM and CM are well known to be highly epileptogenic [2], but the epileptogenicity of MVM remains controversial [3, 4, $5,6]$. The reason for this may be attributed to the complicated pathology of MVM, because MVM is sometimes associated with other epileptogenic vascular malformations [7]. The coexistence of CM and MVM is the most common type of mixed vascular malformation [8], and was reported to occur in up to $33 \%$ of patients with MVM [6]. We operated on an epileptic patient with MVM and CM in the same region, in whom the topographical relationship between the epileptogenic zone and vascular malformations was demonstrated by intraoperative electrocorticography (ECoG), navigation system, and histological findings. The surgical strategy for intractable epilepsy associated with MVM and CM is discussed.

\section{Case Report}

A 53-year-old male was admitted to our hospital because of status epilepticus. At the age of 44 , he developed generalized seizures. At that time, a computed tomographic (CT) scan demonstrated a small intracerebral hematoma in the right frontal lobe, and an an- giogram revealed MVM in the same area. Conservative therapy with anticonvulsants was prescribed and he returned to his daily life without any neurological deficit. He worked in the accounting section of a food company. Since then, he had frequent secondary generalized seizures in spite of optimal dosages of anticonvulsants.

On admission, he was in a drowsy state. EEG showed frequent ictal discharges originating from the right frontal region, which indicated status epileptics. Both T1- and T2weighted magnetic resonance (MR) images showed a mass $18 \mathrm{~mm}$ in diameter with mixed intensity (Fig. 1A). A low-intensity rim was observed around the mass on T2-weighted image. A CT scan demonstrated a high density mass in the right frontal lobe. After administration of contrast medium, linear enhancement was demonstrated at the medial side of the mass (Fig. 1B). Although MR images failed to reveal a linear flow void, an angiogram demonstrated MVM draining from a parasagittal frontal area, depicting the socalled “caput medusae” (Fig. 1C). Fluorodeoxy glucose-positron emission tomography (FDG-PET) demonstrated a hypometabolic anterior frontal lobe, especially the cortex overlying the mass (Fig. 1D). These radiological examinations suggested a de novo CM located just lateral to the known MVM in the right frontal lobe. Ictal EEG and FDG-PET indicated that the anterior frontal lobe was epileptogenic. An angiogram also demonstrated a non-ruptured aneurysm in the right middle cerebral artery (MCA). 
With conservative therapy, the patient became alert without neurological deficit. Prophylactic surgery for right MCA aneurysm and epilepsy surgery were scheduled. Three months after the onset of status epilepticus, right fronto-temporo-parietal craniotomy was performed under intravenous general anesthesia with propofol and fentanyl. After opening the Sylvian fissure, the neck of the aneurysm was successfully clipped. There was no evidence of hemorrhage from the aneurysm.

The cortex of the frontal pole was yellowish, suggesting deposition of hemosiderin. There was a dilated cortical vein on the medial side of the yellowish cortex. Intraoperative ECoG revealed frequent localized spikes over the hemosiderin-deposited cortex (Fig. 2A), which was determined to be above the CM using an intraoperative navigation system (Fig. 2B, C). Anterior frontal lobectomy including the irritative yellowish cortex, CM, and MVM with its tributaries was performed. After the lobectomy, no residual paroxysmal activity was recorded from the surrounding cortex.

The patient became seizure free without any neurological deficits or psychiatric problems after surgery. Histopathological examination of the mass located under the irritative cortex indicated typical CM (Fig. 3A, B). The $\mathrm{CM}$ was surrounded by dilated veins of various sizes (Fig. 3A, C). Dilated veins were noted not only around the CM, but also in the distant cerebral white matter (Fig. 3A). Dilated capillaries in the overlying cortex appeared to drain into these dilated veins. The irritative cortex overlying the CM showed remarkable hemosiderin deposits, gliosis and infiltration of foamy macrophages, probably due to repeated hemorrhagic events from the CM (Fig. 3D).
Fig. 1.

[A] Axial T2-weighted magnetic resonance image demonstrates a mixed intensity mass lesion in the frontal lobe. A hypointense rim is observed around the mass, suggesting hemosiderin deposit. [B] A computed tomography scan demonstrates linear enhancement in the left frontal lobe at the medial side of a high density mass. [C] Lateral view of right carotid angiogram demonstrates an enlarged medullary vein in the right frontal lobe, and several veins draining to the parasagittal frontal cortical vein, forming the so-called "caput medusae". [D] Fluorodeoxy glucose-positron emission tomography (FDG-PET) demonstrates hypometabolism in the anterior frontal lobe, especially in the cortex overlying the mass.




Fig. 2.

[A] Intraoperative electrocorticography over the right frontal lobe demonstrates localized spikes at electrode No. 3 , which is just above the cavernous malformation (CM) on the axial [B] and sagittal views $[\mathrm{C}]$ of the intraoperative navigation system (black arrows).

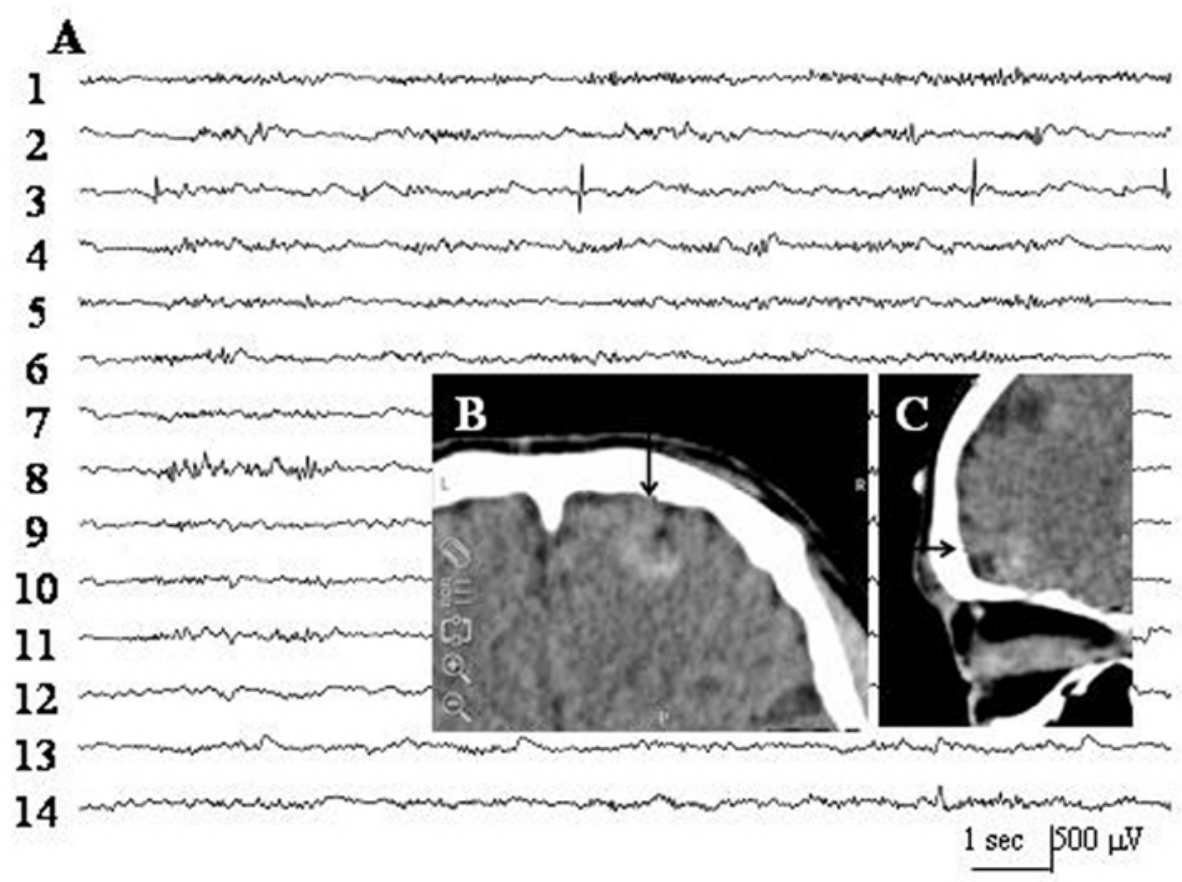

Fig. 3.

[A] Photomicrograph of the anterior frontal lobe [hematoxylin and eosin (H \& E), ×10]. A cavernous malformation (CM) is located just below the irritative cortex (*). The CM is surrounded by variably sized dilated veins of the medullary venous malformation (MVM). Dilated veins are noted not only around the CM, but also in the distant cerebral white matter (arrow). [B] Higher


magnification of the CM indicates closely apposed dilated vascular channels with fibrotic walls (H \& E, $\times 100$ ). [C] The edge of the CM is composed of fibrous tissue, and is surrounded by variably sized dilated veins of the MVM, confirming the radiological findings of "caput medusae". (H \& E, $\times 40$ ) [D]. The irritative cortex overlying the CM shows remarkable hemosiderin deposits, gliosis and infiltration of foamy macrophages, probably due to repeated hemorrhagic events from the CM (glial fibrillary acidic protein staining, $\times 20$ ). 


\section{Discussion}

With the recent advances of neuroimaging techniques, the coexistence of MVM and CM can be determined by imaging studies. Many previous studies $[6,9,10,11,12]$ have reported a close topographical relationship between MVM and CM using contrast-enhanced MR. CMs frequently appear to arise at the distal radicles of MVMs [10, 11]. Although contrast-enhanced MR was not performed in the present case, combined use of precontrast MR and contrast-enhanced CT scans clearly demonstrated that de novo CM [10] was located just medial to the MVM.

Histologically, Rigamonti et al. [13] demonstrated that MVM-related intracranial hemorrhage had an unexpected coexistent CM. However, there was no description of the microscopic relationship between MVM and $\mathrm{CM}$. The main reason is that when craniotomy is performed in patients with MVM and CM, every attempt is made to spare the MVM in order to avoid the risk of venous infarction [9, 14, 15], because the MVM participates in compensatory venous drainage [6].

The only exception in which a surgical specimen containing both CM and MVM is likely to be obtained is where a large portion of the epileptogenic zone is being excised simultaneously for intractable epilepsy, such as in the present case. In such cases, resection of part or all of the MVM may be justified [9]. However, as far as we know, there has been no previous report on the microscopic relationship between CM and MVM. Awad et al. [9] reported two epileptic patients in whom zones of capillary telangiectasis in the surrounding brain parenchyma appeared to coalesce with the CM at its periphery. In our case, the CM was surrounded by many dilated veins of the MVM, supporting the radiological findings that CM frequently appear to arise at the distal radicles of MVM, hence the name caput medusae.

Although the epileptogenicity of MVM remains controversial $[3,4,5,6]$, we demonstrated in a previous report that supratentorial MVM itself is not epileptogenic and an associated CM may be highly epileptogenic [4]. In the present case, intraoperative ECoG together with an intraoperative navigation system demonstrated that the irritative area was located within the yellowish cortex overlying the CM. Histological examination of this cortex revealed hemosiderin deposit and astrogliosis, indicating that the epileptogenicity of this patient is due to repeated hemorrhage from the CM.

In surgical treatment for epilepsy associated with CM and MVM, it is generally considered that excising the $\mathrm{CM}$ and the surrounding epileptogenic cortex that contains hemosiderin and gliosis is sufficient, while the main trunk of the MVM may be preserved as the MVM itself is not epileptogenic [4]. However, some reports have raised the question of whether the abnormal draining vein of the MVM is the actual disease site that induces blood flow disturbances, which in turn results in the formation of a CM (de novo and recurrent) $[8,9]$. Thus, resection of the MVM in addition to removal of the CM and surrounding epileptogenic cortex is desirable, when the MVM and its tributaries are located 
in the non-eloquent area. In the present case, since the MVM and CM were located in the non-eloquent right frontal lobe, anterior frontal lobectomy including the irritative cortex, CM, MVM and its tributaries was performed.

As far as we know, this is the first reported case of surgical treatment for MVM, CM, and aneurysm in the same craniotomy. However, we speculated that the coexistence of the vascular malformation and aneurysm was incidental because the feeding artery of the vascular malformation and the parent artery of the aneurysm were different. Furthermore, the unruptured MCA aneurysm was not epileptogenic according to the intraoperative ECoG. Thus, we performed prophylactic neck clipping for the right MCA aneurysm, followed by the epilepsy surgery to remove the MVM and $\mathrm{CM}$ including the epileptic focus.

\section{References}

[1] Russell DS, Rubinstein LJ. Pathology of Tumors of the Central Nervous System. London: Edward Arnold, 1951.

[2] Kraemer DL, Awad IA. Vascular malfomations and epilepsy: clinical considerations and basis mechanisms. Epilepsia 1994;35 (Suppl 6):S30-S43.

[3] Garner TB, Del Curling O Jr, Kelly DL Jr, Laster DW. The natural history of intracranial venous angioma. J Neurosurg 1991;75:715-722.

[4] Morioka T, Hashiguchi K, Nagata S, Miyagi Y, Yoshida F, Mihara F, Sakata A, Sasaki T. Epileptogenicity of supratentorial medullary venous malformation. Epilepsia 2006;47:365-370.

[5] Striano S, Nocerino C, Striano P, Boccella
P, Meo R, Bilo L, Cirillo S. Venous angioma and epilepsy. Neurol Sci 2000;21:151-155.

[6] Topper R, Jurgens E, Reul J, Thron A. Clinical significance of intracranial developmental venous anomalies. J Neurol Neurosurg Psychiatry 1999;67:234-238.

[7] Abdulrauf SI, Kaynar MY, Awad IA. A comparison of the clinical profile of cavernous malformations with and without associated malformations. Neurosurgery 1999;44:41-47.

[8] Perrini P, Lanzino G. The association of developmental anomalies and cavernous malformations: pathophysiological, diagnostic, and surgical considerations. Neurosurg Focus 2006;21:e5.

[9] Awad IA, Robinson JR Jr, Mohanty S, Estes ML. Mixed vascular malformations of the brain: clinical and pathogenetic considerations. Neurosurgery 1993;33:179-188.

[10] Campeau NG, Lane J. De novo development of a lesion with the appearance of a cavernous malformation adjacent to an existing developmental venous anomaly. AJNR Am J Neuroradiol 2005;26:156-159.

[11] Dillon WP: Cryptic vascular malformations: controversies in terminology, diagnosis, pathophysiology, and treatment. AJNR Am J Neuroradiol 1997;18:1839-1846.

[12] Wilms G, Bleus E, Demaerel P, Marchal G, Plets C, Goffin J, Baert AL. Simultaneous occurrence of developmental venous anomalies and cavernous angiomas. AJNR Am J Neuroradiol 1994;15:1247-1257.

[13] Rigamonti D, Spetzler RF, Medina M, Rigamonti K, Geckle DS, Pappas C. Cerebral venous malformations. J Neurosurg 1990;73:560-564.

[14] Miyagi Y, Mannoji H, Akaboshi K, Morioka T, Fukui M: Intraventricular cavernous 
malformation associated with medullary venous malformation. Neurosurgery 1993;32:461-464.

[15] Sasaki O, Tanaka R, Koike T, Koide A, Koizumi T, Ogawa H: Excision of cavernous angiomas with preservation of coexisting venous angioma. J Neurosurg 1991;75:461-464. 